\title{
Rapid, quantitative reverse transcriptase-polymerase chain reaction: Application to intraoperative molecular detection of occult metastases in esophageal cancer
}

\author{
Siva Raja, BS ${ }^{a}$ \\ James D. Luketich, MDa \\ Lori A. Kelly, BS \\ William E. Gooding, MS ${ }^{b}$ \\ Sydney D. Finkelstein, MDC \\ Tony E. Godfrey, PhDa
}

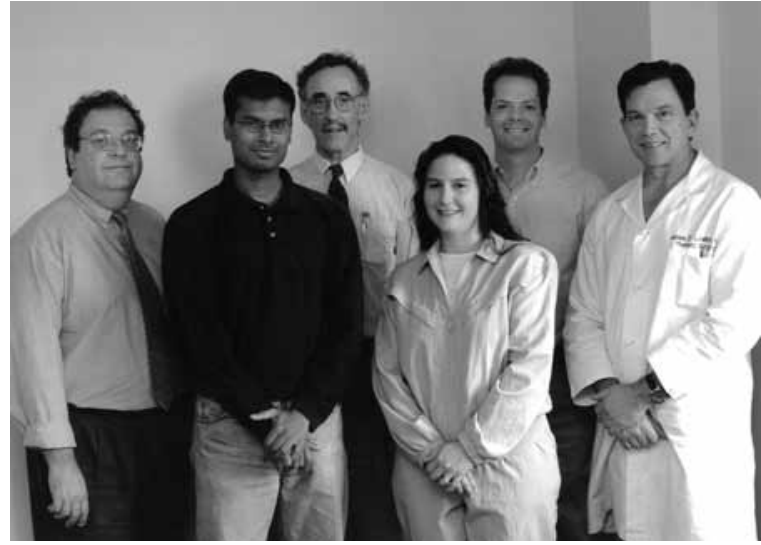

Finkelstein, Raja, Gooding, Kelly, Godfrey, Luketich (left to right)

Objective: Our earlier data showed that quantitative reverse transcriptase-polymerase chain reaction can discriminate patients with node-negative cancer who are at high risk for recurrence. The objective of this study was to determine whether a new, more rapid quantitative reverse transcriptase-polymerase chain reaction assay could provide this information in a time frame suitable for intraoperative decision making.

Methods: We studied formalin-fixed, archived lymph nodes from 30 patients with histologically determined node-negative esophageal cancer with rapid quantitative reverse transcriptasepolymerase chain reaction to measure expression of carcinoembryonic antigen messenger RNA. We also performed rapid quantitative reverse transcriptase-polymerase chain reaction on 37 snap-frozen lymph nodes from 23 patients. Eleven of the 23 patients had benign esophageal disorders (negative control group). The other 12 had esophageal cancer, 6 with histologically determined positive lymph nodes and 6 with histologically determined negative lymph nodes.

From the Departments of Surgery, ${ }^{\text {a }}$ Biostatistics, ${ }^{\mathrm{b}}$ and Pathology, ${ }^{\mathrm{c}}$ University of Pittsburgh Medical Center, Pittsburgh, Pa.

Supported by National Institutes of Health National Cancer Institute research grant CA90665-01.

Read at the Eighty-first Annual Meeting of The American Association for Thoracic Surgery, San Diego, Calif, May 6-9, 2001.

Received for publication May 5, 2001; revisions requested June 27, 2001; revisions received Aug 16, 2001; accepted for publication Aug 30, 2001.

Address for reprints: Division of Thoracic Surgery, Suite C-800 Presbyterian University Hospital, 200 Lothrop St, Pittsburgh, PA 15213.

J Thorac Cardiovasc Surg 2002;123:475-83

Copyright (c) 2002 by The American Association for Thoracic Surgery.

0022-5223/2002 $\$ 35.00+0 \quad \mathbf{1 2 / 6 / 1 1 9 8 8 4}$

doi:10.1067/mtc.2002.119884
Results: In the retrospective analysis of archival tissue from 30 patients with esophageal cancer with histologically determined negative lymph nodes, rapid quantitative reverse transcriptase-polymerase chain reaction predicted disease recurrence with a sensitivity and a specificity of $90 \%$ and $80 \%$, respectively, and was comparable to conventional quantitative reverse transcriptase-polymerase chain reaction. In the frozen-tissue analysis rapid quantitative reverse transcriptasepolymerase chain reaction detected significantly higher levels of carcinoembryonic antigen expression in all 12 of the histologically determined positive lymph nodes than in the benign nodes. For 2 of these 12 nodes the intraoperative frozen-section analysis had negative histologic results, and N1 status was determined only on final pathologic examination. Rapid (intraoperative) quantitative reverse transcriptasepolymerase chain reaction discriminated both nodes as positive. Among the 14 histologically determined negative nodes, 1 of 3 nodes from 1 patient showed increased carcinoembryonic antigen according to rapid quantitative reverse transcriptase-polymerase chain reaction, and this patient had a clinical recurrence.

Conclusions: In our study we were able to rapidly discriminate patients with node negative-esophageal cancer who had a high risk of recurrence. In frozen tissues rapid quantitative reverse transcriptase-polymerase chain reaction correlated with 


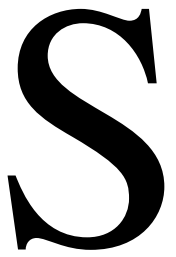

urgical decisions in the treatment of esophageal cancer and other malignancies are often based on intraoperative frozen-section analysis of lymph nodes. The 5-year survival for patients with esophageal cancer remains poor, at $5 \%$ to $10 \%$, because of the presence of advanced disease in many patients at initial presentation. ${ }^{1}$ If local and regional lymph nodes are histologically negative, there is a dramatic improvement in the 5-year survival. Nevertheless, $30 \%$ to $50 \%$ of patients with histologically node-negative disease have recurrence. ${ }^{2,3}$ This is most likely because of the limitations of current staging techniques in the detection of micrometastases. As a result, other techniques, such as immunohistochemical examination or reverse transcriptase-polymerase chain reaction (RT-PCR), have been used in attempts to detect histologically occult micrometastases. ${ }^{4-7}$

Studies on esophageal cancer, ${ }^{6}$ colon cancer, ${ }^{8}$ melanoma, ${ }^{9,10}$ and breast cancer ${ }^{11,12}$ have shown that RTPCR can detect histologically occult micrometastases and may predict recurrence. These data have been criticized, however, because of false-positive results in control samples and the subsequent low specificity and positive predictive value of the RT-PCR assay. Recently we showed that quantitative RT-PCR allows the distinction of background or ectopic gene expression from true micrometastases and can therefore avoid false-positive results and increase specificity in predicting disease recurrence. ${ }^{13}$ Our goal now is to be able to provide the surgeon with this critical information at a time when important surgical decisions are made, during the operation.

In this study we report on the development and testing of an extremely rapid quantitative RT-PCR assay that can be carried out in less than 30 minutes. To validate the rapid assay we compared it with standard quantitative RT-PCR and correlated results with clinical outcomes among patients with esophageal cancer.

\section{Material and Methods Patients}

For the retrospective analysis we studied lymph nodes from 30 patients (Table 1) who underwent curative resection for histologically determined node-negative esophageal cancer. All operations were performed in the Division of Thoracic Surgery at the University of Pittsburgh Medical Center between 1991 and 1998. Clinical follow-up was obtained from the medical records and was confirmed for all patients as of August 2001. The median clinical follow-up time for all patients was 36 months; that for the surviving patients was 49 months (range 28-91 months).

For the prospective analysis lymph node samples were obtained from 12 patients undergoing either staging or resection for esophageal cancer identified by the Section of Thoracic Surgery at the University of Pittsburgh Medical Center from 1999 to 2000 (Table 2). Control nodes from patients without cancer were obtained incidentally from patients undergoing abdominal surgery for antireflux procedures. At the time of excision half of each lymph node was frozen in liquid nitrogen for analysis, and the remainder was sent for routine pathologic analysis. The excised tissue was removed as part of the routine clinical course at our institution, and no additional tissue was removed for any purely research purpose. All tissues were collected as part of an ongoing, institutional review board-approved Esophagus Cancer Risk Registry protocol at the University of Pittsburgh.

\section{Tissue and RNA Isolation}

All tissues used in the retrospective study were formalin-fixed, paraffin-embedded archival specimens obtained from the pathology tissue banks. Hematoxylin and eosin-stained slides were also retrieved for each tissue block and were examined to confirm the original node-negative diagnosis. Tissue blocks were mounted on a microtome, and 5 to 15 sections $5.0 \mu \mathrm{m}$ thick were cut and placed in $2.0 \mathrm{~mL}$ ribonuclease-free tubes. At the same time $2 \mathrm{sec}-$ tions $5.0 \mu \mathrm{m}$ thick were cut (first and last sections) and mounted on microscope slides for hematoxylin and eosin staining and also immunohistochemical staining with antibodies against carcinoembryonic antigen (CEA). RNA was isolated by means of previously described methods ${ }^{14}$ and quantitated spectrophotometrically.

The fresh-frozen lymph node tissues were embedded in Optimal Cutting Temperature compound and 10 to 15 sections 4.0 $\mu \mathrm{m}$ thick were cut. At the same time 2 sections $4.0 \mu \mathrm{m}$ thick were cut (first and last sections) and mounted on microscope slides for hematoxylin and eosin staining. The remaining sections were placed in $1.5 \mathrm{~mL}$ ribonuclease-free tubes with the lysis buffer from the RNeasy Mini kit (QIAGEN Inc, Valencia, Calif). RNA was extracted according to the manufacturer-recommended protocol with the following modifications. Centrifugation times longer than 1 minute were reduced to 1 minute, and the RNA was reconstituted in $60 \mu \mathrm{L} \mathrm{RNA}$ secure resuspension solution (Ambion Inc, Austin, Tex). Although most of the samples were processed together, 5 
TABLE 1. Clinical characteristics of the study population

\begin{tabular}{|c|c|c|c|}
\hline \multirow[b]{2}{*}{ Characteristic } & \multirow[b]{2}{*}{ Patients $(\mathrm{n}=\mathbf{3 0}$ ) } & \multicolumn{2}{|c|}{ Quantitative RT-PCR result } \\
\hline & & Negative $(n=17)$ & Positive ( $n=13$ ) \\
\hline \multicolumn{4}{|l|}{ Sex } \\
\hline Male & 22 & 14 & 8 \\
\hline Female & 8 & 3 & 5 \\
\hline \multicolumn{4}{|l|}{ Follow-up (mo) } \\
\hline Median & 36 & 43.9 & 31.8 \\
\hline Range & $5-90.5$ & $5-90.5$ & 6.3-75.6 \\
\hline Mean age at diagnosis (y) & 68.3 & 68.4 & 68.2 \\
\hline \multicolumn{4}{|l|}{ Tumor type } \\
\hline Adenocarcinoma & 26 & 16 & 10 \\
\hline Squamous cell & 4 & 1 & 3 \\
\hline \multicolumn{4}{|l|}{ Stage $^{*}$} \\
\hline $0 \dagger$ & 1 & 1 & 0 \\
\hline I & 10 & 8 & 2 \\
\hline IIA & 15 & 8 & 7 \\
\hline Median number of nodes examined & 12.5 & 12 & 15 \\
\hline
\end{tabular}

*Four patients who received chemotherapy had no tumor at operation.

tPatient had cancer on biopsy but not at resection.

samples were processed individually to determine the median extraction time per sample. The RNA yield and purity were determined spectrophotometrically for quality control purposes.

\section{Quantitative RT-PCR}

Quantitative RT-PCR was carried out with the 5'-nuclease assay. ${ }^{15,16}$ Rapid quantitative RT-PCR was carried out on the Cepheid Smart Cycler real-time DNA amplification and detection system (Cepheid, Sunnyvale, Calif) as described here. Standard quantitative RT-PCR was carried out on the Applied Biosystems 7700 Sequence Detection Instrument (TaqMan) with a single-tube quantitative RT-PCR procedure as described previously elsewhere. ${ }^{17}$

\section{Rapid quantitative RT-PCR}

Expression of the $C E A$ gene was measured relative to that of the endogenous control gene, the $\beta$-glucuronidase gene (GUSB) with the comparative threshold cycle method described previously elsewhere. ${ }^{14,18}$ All quantitative RT-PCR assays were carried out in triplicate on $400 \mathrm{ng}$ of total RNA. Both CEA and GUSB PCR primers were designed and tested to avoid amplification of genomic DNA. However, control reactions were still run with RNA without reverse transcription (no transcription control) and water (no template control) in place of complementary DNA as PCR template. Together these control reactions ruled out the possibility that any signal was generated from either genomic DNA contamination of the RNA or PCR product contamination of the reagents. In addition, a calibrator RNA sample was amplified in parallel on all runs to allow comparison of samples run at different times ${ }^{14,19}$ and to determine reproducibility of the assay.

The final concentrations of the reaction components were as follows: 1× PCR Platinum Taq buffer (Life Technologies, Inc, Rockville, Md), 300-nmol/L concentrations of each deoxyribonu-
TABLE 2. Tumor stage and histologic status for patients in the rapid quantitative RT-PCR study on fresh-frozen lymph nodes

\begin{tabular}{lll}
\hline Patient & Stage & Tumor type \\
\hline 12 & IIA & Adenocarcinoma \\
13 & IIA & Adenocarcinoma \\
14 & I & Adenocarcinoma \\
15 & IIA & Adenocarcinoma \\
16 & IIA & Adenocarcinoma \\
17 & IIA & Squamous cell \\
18 & III & Adenocarcinoma \\
19 & III & Adenocarcinoma \\
20 & III & Adenocarcinoma \\
21 & III & Adenocarcinoma \\
22 & III & Adenocarcinoma \\
23 & III & Adenocarcinoma
\end{tabular}

cleoside triphosphate, $4.5-\mathrm{mmol} / \mathrm{L}$ magnesium chloride, $0.8-\mathrm{U} / \mu \mathrm{L}$ ribonuclease inhibitor, $1.25-\mathrm{U} / \mu \mathrm{L}$ Sensiscript reverse transcriptase (QIAGEN), 0.06-U/ $\mu \mathrm{L}$ Platinum Taq, 60-nmol/L reverse transcription primer, 400-nmol/L concentrations of each PCR primer, 200$\mathrm{nmol} / \mathrm{L}$ probe, and $400 \mathrm{ng}$ total RNA. The total RNA input for the fresh-tissue analysis was $5 \mu \mathrm{L}$ of the sample. For the fixed-tissue analysis $5 \mu \mathrm{L}$ of an $80-\mathrm{ng} / \mu \mathrm{L}$ dilution of the RNA stock was used. In the rapid assay the reverse transcription reaction was carried out without the PCR primers or probe, then the tube was opened and the primers and probe were added. This was necessary because a standard single-step quantitative RT-PCR lacked the sensitivity to detect rare messages. ${ }^{17}$ The oligonucleotide sequences used were as follows: GUSB reverse transcription primer, 5'-TGG TTG TCT 


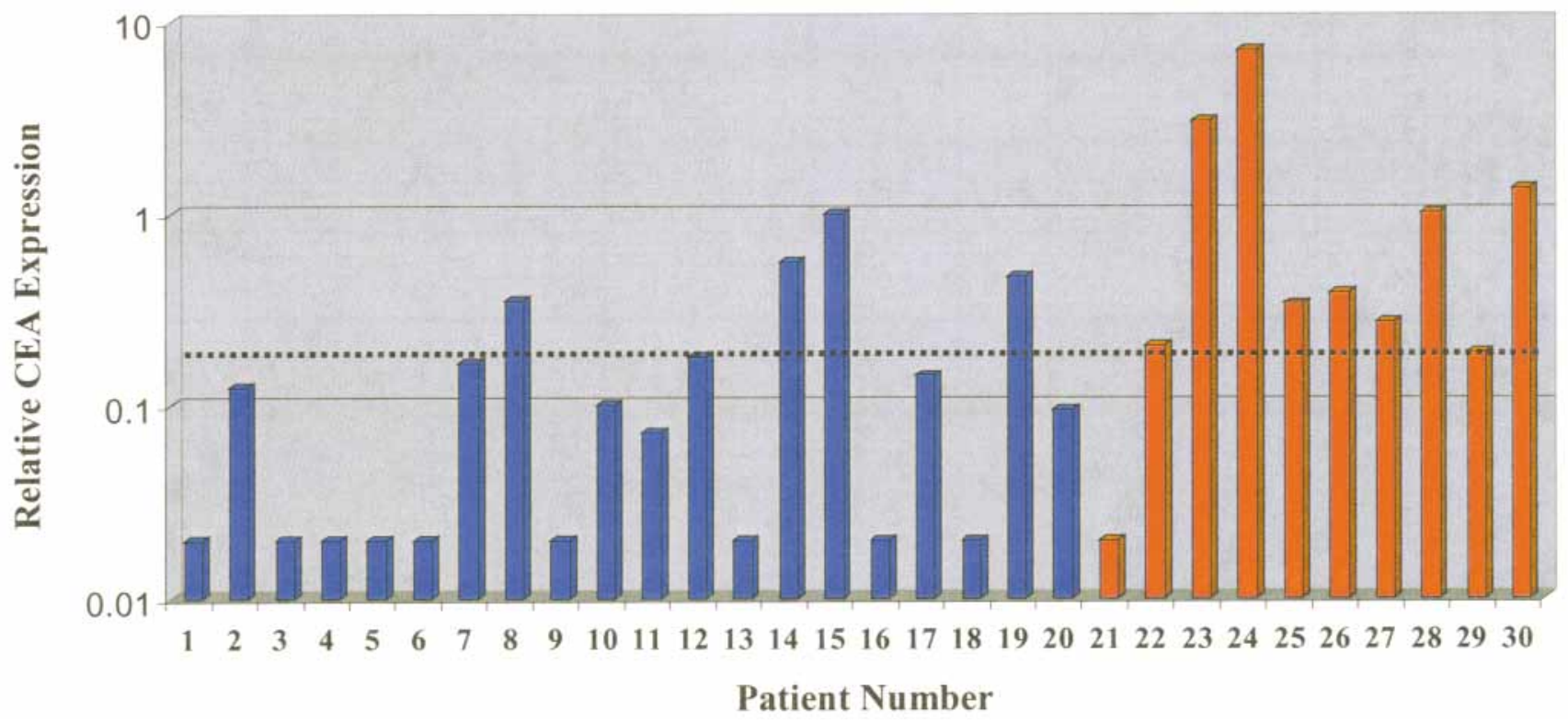

Figure 1. Smart Cycler analysis of CEA expression in formalin-fixed lymph nodes from 30 patients with pathologically determined node-negative esophageal cancer. Red bars represent patients with recurrence; blue bars represent patients without recurrence. Lymph nodes with no detectable CEA expression were arbitrarily plotted at 0.02 . Dashed line indicates CEA expression cutoff value (0.183) most accurate for predicting disease recurrence.

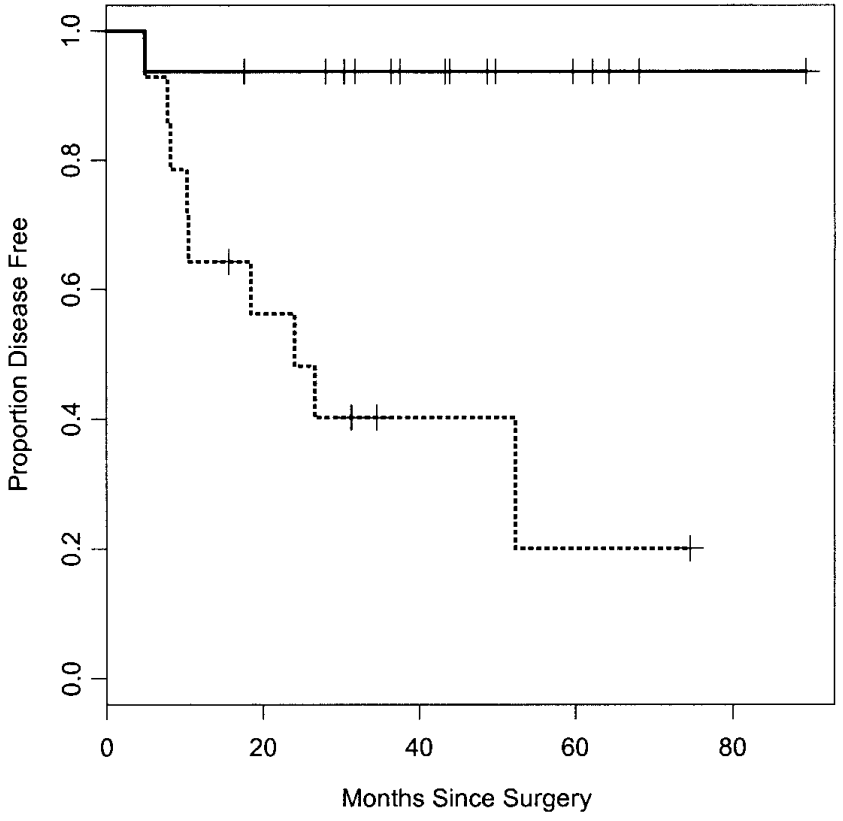

Figure 2. Kaplan-Meier disease-free survival curve for 30 patients with pNO esophageal cancer restratified as node-positive (dashed line) or node-negative (solid line) because of values above or below cutoff value of $\mathbf{0 . 1 8 3}$ according to Smart Cycler quantitative RT-PCR.

CTG CCG A-3’; GUSB forward PCR primer, 5'-CTC ATT TGG AAT TTT GCC GAT T-3’; GUSB reverse PCR primer, 5'-CCG AGT GAA GAT CCC CTT TTT A-3'; GUSB probe 5'-VIC TGA
TABLE 3. Rapid quantitative RT-PCR results on 30 patients with pNO esophageal cancer with a CEA expression cutoff of 0.183

\begin{tabular}{lcc}
\hline $\begin{array}{l}\text { Quantitative } \\
\text { RT-PCR result }\end{array}$ & $\begin{array}{c}\text { Recurrence } \\
\text { (true positive) }\end{array}$ & $\begin{array}{c}\text { No recurrence } \\
\text { (true negative) }\end{array}$ \\
\hline Positive & 9 & 4 \\
Negative & 1 & 16
\end{tabular}

Sensitivity was $90 \%(9 / 10)$, and specificity was $80 \%(16 / 20)$.

ACA GTC ACC GAC GAG AGT GCT GG-6-carboxyl-tetramethyl-rhodamine-3' (VIC, Applied Biosystems, Foster City, Calif); $C E A$ reverse transcription primer, 5'-GTG AAG GCC ACA GCA T-3'; $C E A$ forward PCR primer, 5'-AGA CAA TCA CAG TCT CTG CGG A-3'; $C E A$ reverse PCR primer, 5'-ATC CTT GTC CTC CAC GGG TT-3'; and CEA probe, 5'-6-carboxyfluorescein CAA GCC CTC CAT CTC CAG CAA CAA CT-6-carboxytetramethyl-rhodamine-3'. Rapid quantitative RT-PCR reactions were carried out on the Cepheid Smart Cycler with the following thermocycler conditions: $48^{\circ} \mathrm{C}$ hold for 5 minutes, $70^{\circ} \mathrm{C}$ for $60 \mathrm{sec}-$ onds (for the addition of the PCR primers and probe), $95^{\circ} \mathrm{C}$ hold for 30 seconds (for Platinum Taq activation), and 45 cycles of $95^{\circ} \mathrm{C}$ for 2 seconds and $64^{\circ} \mathrm{C}$ for 15 seconds. Data were analyzed by means of Smart Cycler software (version 1.0) with the second derivative method for determining the threshold. For the fixed-tissue analysis a 30-minute reverse transcription reaction was required because of the reduced sensitivity associated with the degradation inherent in fixed-tissue RNA samples. ${ }^{14}$ 


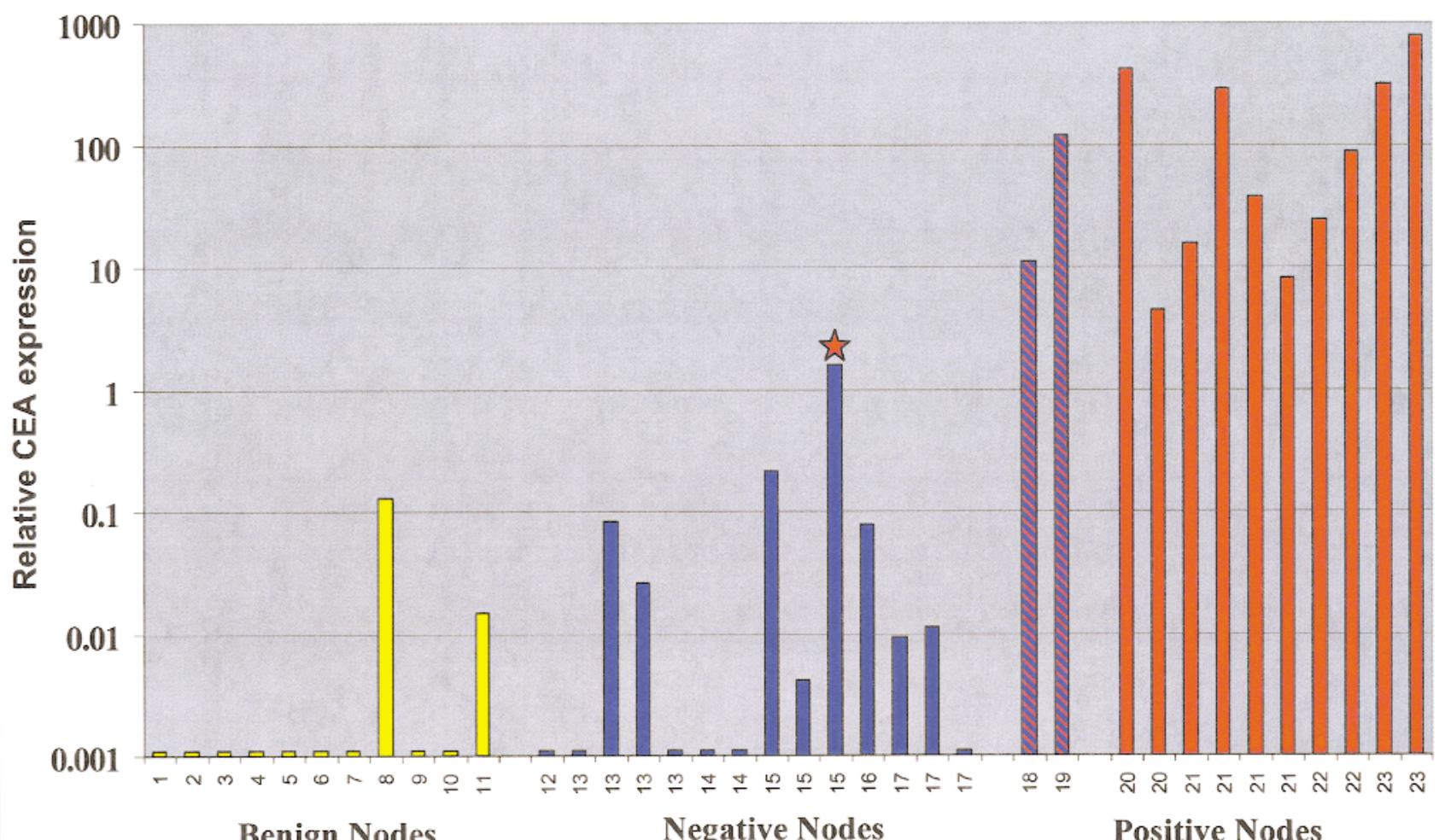

Figure 3. Smart Cycler analysis of CEA expression in frozen lymph nodes. Yellow bars represent nodes from patients without cancer; blue bars represent nodes determined on final pathologic examination to be negative for disease; red bars represent nodes found to be positive for disease; striped bars represent nodes that were negative according to intraoperative frozen-section analysis but positive according to fixed-tissue histologic examination; red star represents a highly expressing node from patient with node-negative disease who had recurrence. Samples with no detectable CEA expression were arbitrarily plotted at 0.001 .

\section{Statistical Analysis}

The predictive validity of rapid quantitative RT-PCR determination of CEA expression level was evaluated by proportionate hazards regression for disease-free survival. Disease-free survival was defined as the time from the operation to diagnosis of recurrent esophageal cancer. Deaths from other causes and patients alive without disease as of August 1, 2001, were censored. CEA expression was also used to classify patients as having either positive or negative results of quantitative RT-PCR. The cutoff level for classification, as determined by receiver operating characteristic curve analysis, ${ }^{20,21}$ was defined as the CEA expression level value that produced the most accurate classification with disease recurrence as the standard. Sensitivity and specificity of quantitative RT-PCR results for diagnosing occult metastasis were calculated. Patients classified as having positive or negative quantitative RT-PCR results according to the classification method were tested for differences in disease-free survival with the log-rank test.

\section{Results}

\section{Archived Tissue Analysis}

Initially we analyzed archived, histologically determine negative lymph nodes (N0) from 30 patients with esophageal cancer (Figure 1). Of the 30 tissue blocks analyzed, 13 had CEA expression higher than the receiver operating characteristic curve-determined cutoff $(>0.183)$. Of this group, 9 patients had disease recurrence and had died by the end of the study, 2 had died of other causes by the end of the study, and 2 were alive without disease at the end of the study. Of the remaining 17 patients with negative quantitative RT-PCR results, 1 had disease recurrence at 5 months and had died by the end of this study, 5 had died of other causes by the end of the study, and 11 remained alive and disease free at the end of the study (Table 3). The sensitivity and specificity for predicting disease recurrence of the rapid quantitative RT-PCR assay were $90 \%$ and $80 \%$, respectively, and $83 \%$ of patients in the cohort were classified correctly. Figure 2 shows the Kaplan-Meier diseasefree survival curves for patients with positive and negative quantitative RT-PCR results. Survivals at 5 years among patients with negative and positive quantitative RT-PCR results were $94 \%$ and $20 \%$, respectively. The survival functions for these two groups were statistically significantly different $(P=.001$ by log-rank test). 
According to the proportionate hazards model for disease-free survival, the relative risk associated with an increase of 1 unit of CEA expression was 1.52 (95\% confidence interval 1.11-2.09, $P=.027)$. That associated with a CEA expression greater than 0.183 was 3.78 (95\% confidence interval $1.34-10.7, P=.0007$ ) relative to CEA expression of 0.183 or less.

\section{Fresh-Tissue Analysis}

Fresh-frozen sections of lymph nodes from patients undergoing minimally invasive staging for esophageal cancer or benign esophageal disorders were analyzed. In the 11 lymph nodes from the benign cases, rapid quantitative RTPCR detected extremely low levels of CEA expression in only 2 lymph nodes (patients 8 and 11, Figure 3 ). This low level in the benign cases presumably represented background or ectopic CEA expression. The highest level of CEA expression in any benign node was 34-fold lower than the lowest-expressing positive node (Figure 3), which had only a small focus of tumor involvement.

Of the 26 lymph nodes from 12 patients with esophageal cancer, 12 nodes from 6 patients were ultimately shown to be positive on final pathologic examination. In 2 of these nodes from 2 patients, however, results of intraoperative frozen-section analysis were negative, and N1 status was determined after the operation. Rapid quantitative RT-PCR showed CEA expression levels in these 2 nodes to be in the range of the histologically determined positive nodes (patients 18 and 19, Figure 3). Among the 6 patients who had histologically determined node-negative esophageal cancer, only one had a single node with increased CEA expression according to rapid quantitative RT-PCR (patient 15 , Figure 3 ). This patient had a clinical recurrence of his esophageal cancer at a follow-up of 16.5 months.

Analysis of the calibrator samples on each run revealed that for 13 separate runs the SD of the change in threshold cycle value was 0.14 cycles (approximately $10 \%$ of the SD for relative expression measurements). In addition, the quantitative RT-PCR required approximately 25 minutes for all 40 cycles. With an RNA isolation time of 5 to 7 minutes, the entire assay took approximately 30 minutes. In our hands this was an extremely rapid and reproducible assay.

\section{Discussion}

In many malignancies, including esophageal cancer, TNM staging yields the best prognostic information. The absence of nodal involvement is associated with a more localized lesion and suggests that a cure may be obtained by surgical resection alone. Nodal metastases, in contrast, are indicators of tumor spread beyond the site of the primary tumor and as such necessitate systemic treatment. Although the role of chemotherapy in esophageal cancer remains controversial, some trials have shown trends indicating the bene- fit of preoperative chemotherapy. ${ }^{22,23}$ One advantage of this approach is that more patients are able to complete the chemotherapy protocol than would be able do so if they were to receive treatment after a surgical procedure that carries a high morbidity. In the near future ongoing prospective, randomized clinical trials should resolve the utility of preoperative chemotherapy in patients with esophageal cancer. With the advent of minimally invasive surgical approaches to staging, there is a potential for staging before resection, which allows the offer of preoperative chemotherapy when appropriate. The accurate determination of nodal stage before attempted curative resection therefore could become extremely important in the decision-making process for patients with esophageal cancer.

Currently, intraoperative nodal staging is performed by frozen sectioning and hematoxylin and eosin staining. Although this methodology has at least a 93\% correlation with the criterion standard of formalin-fixed, hematoxylin and eosin-stained tissue examination, ${ }^{24}$ both methods have significant limitations. Specifically, $30 \%$ to $50 \%$ of patients with histologically determined node-negative esophageal cancer have disease recurrence despite potentially curative resection. This finding is most likely the result of the sampling error that is inherent in the conventional methodologies and of a lack of sensitivity for detecting micrometastases. Single tumor cells or small foci of cells are easily missed, because only a couple 4- $\mu \mathrm{m}$ sections are studied, with the vast majority of each node remaining unexamined. Serial sectioning of lymph nodes can reduce the sampling error and thus minimize this limitation. For example, in studies on breast cancer, serial sectioning of lymph nodes combined with immunohistochemical methods led to upstaging of an additional $10 \%$ to $23 \%$ of nodes that were negative according to routine histopathologic evaluation. ${ }^{25,26}$ This method is not routinely used, however, because of the significant time and labor involved in serial sectioning of numerous lymph nodes from each case.

In the last decade many studies have used RT-PCR to detect tumor-related messenger RNA in lymph nodes in attempts to improve the sensitivity of micrometastasis detection. Studies on several cancer types have reported that RTPCR is a reasonable prognostic indicator, ${ }^{8-10}$ but despite this RT-PCR has not made its way into clinical practice. The main reasons for this are poor specificity $(40 \%-60 \%), 8,9$ false-positive results in control nodes from patients without cancer, ${ }^{7}$ and the lack of standardized assays for multicenter trials. In previous work we showed that quantitative RT-PCR can overcome false-positive results from background or ectopic gene expression and can increase the specificity for predicting disease recurrence. ${ }^{13}$ With the techniques used in that study, however, the RNA isolation and RT-PCR assay requires 4 to 6 hours to complete. As a result, this method can only be used after the operation. Intraoperative quantita- 
tive RT-PCR will work only if it can be performed rapidly enough to yield a result in a time frame comparable to that of intraoperative frozen-section analysis (approximately 2030 minutes). Along with rapid RNA isolation and reverse transcription protocols, this requires extremely fast polymerase chain reaction ramping times, such as those attainable with the Roche Light Cycler or the Cepheid Smart Cycle. In this study we used the Caught instrument because of the relative ease of use (no glass capillaries), the independent control of each reaction site, the availability of 4-color multiplexing, and the potential for automated sample preparation and quantitative RT-PCR. With this instrument we have shown that a quantitative RT-PCR assay can be performed on OCT-embedded tissue sections within 30 minutes from RNA extraction to result. With modifications to the protocol reported here, our current, fastest quantitative RTPCR can be carried out in 18.5 minutes (data not shown).

With our rapid assay we performed a retrospective analysis of RNA from archival tissues with a median patient follow-up of 36 months. Our data showed that the rapid assay predicted disease recurrence with a sensitivity and specificity of $90 \%$ and $80 \%$, respectively. These data were comparable with the slower, and more traditional, TaqMan-based analysis $(90 \%$ and $90 \%)$. In our fresh-tissue analysis, we were clearly able to distinguish all the histologically determined positive nodes from the benign control nodes in less than 30 minutes. On the basis of the expression level of CEA, it was also possible to characterize pathologically negative nodes as having an expression pattern that resembled either benign or pathologically positive nodes. From our data, it appears that rapid quantitative RT-PCR is at least as sensitive as fixed-tissue examination and is more sensitive than intraoperative frozen section examination. This is demonstrated by the 2 positive nodes that were deferred or misdiagnosed during the intraoperative examination. To confirm this data, we are planning to study a much larger number of fresh lymph nodes and correlate the results with pathologic staging. As the data set matures, we will also correlate quantitative RT-PCR with recurrence. This should allow us to set an optimal CEA expression cutoff value in the fresh-tissue data set, because it may be different from the cutoff for archived tissues. Because of our promising preliminary fresh-tissue data and problems inherent in analysis of archived tissues, we are hopeful that specificity and positive predictive values will improve with analysis of fresh tissues.

We realize that further development of this technology will be necessary to bring intraoperative quantitative RTPCR from bench to bedside. In a clinical setting the quantitative RT-PCR assay has to be a simple and automated process, with minimal handling. Toward this end our goal is to develop a cartridge-based processor for automated analysis. We envision that the end product will be a single-use disposable cartridge capable of both RNA isolation and quantitative RT-PCR setup. When integrated with a rapidly cycling, real-time quantitative thermal cycle, this system will provide a quantitative RT-PCR result from frozen sections in less than 25 minutes. With the identification of new and accurate markers, this technology could also be used for molecular analysis of surgical margins and as an adjunct to fine-needle aspirate cytologic examination. Thus molecular information regarding micrometastases and completeness of resection could for the first time be made available to the surgeon during the operation. Furthermore, the availability of an automated and reproducible quantitative RTPCR format would allow the true molecular diagnostic value of quantitative RT-PCR to be evaluated in standardized multicenter trials.

\section{References}

1. Silverberg E, Boring CC, Squires TS. Cancer statistics, 1990. CA Cancer J Clin. 1990;40:9-26.

2. Steup WH, De Leyn P, Deneffe G, Van Raemdonck D, Coosemans W, Lerut T. Tumors of the esophagogastric junction: long-term survival in relation to the pattern of lymph node metastasis and a critical analysis of the accuracy or inaccuracy of pTNM classification. $J$ Thorac Cardiovasc Surg. 1996;111:85-94.

3. Akiyama H, Tsurumaru M, Udagawa H, Kajiyama Y. Radical lymph node dissection for cancer of the thoracic esophagus. Ann Surg. 1994;220:364-72.

4. Izbicki JR, Hosch SB, Pichlmeier U, Rehders A, Busch C, Niendorf A, et al. Prognostic value of immunohistochemically identifiable tumor cells in lymph nodes of patients with completely resected esophageal cancer. N Engl J Med. 1997;337:1188-94.

5. Kijima F, Natsugoe S, Takao S, Aridome K, Baba M, Yoshifumi M, et al. Detection and clinical significance of lymph node micrometastasis determined by reverse transcription-polymerase chain reaction in patients with esophageal carcinoma. Oncology. 2000;58:38-44.

6. Luketich JD, Kassis ES, Shriver SP, Nguyen NT, Schauer PR, Weigel TL, et al. Detection of micrometastases in histologically negative lymph nodes in esophageal cancer. Ann Thorac Surg. 1998;66:1715-8.

7. Bostick PJ, Chatterjee S, Chi DD, Huynh KT, Giuliano AE, Cote R, et al. Limitations of specific reverse-transcriptase polymerase chain reaction markers in the detection of metastases in the lymph nodes and blood of breast cancer patients. J Clin Oncol. 1998;16:2632-40.

8. Liefers GJ, Cleton-Jansen AM, van de Velde CJ, Hermans J, van Krieken JH, Cornelisse CJ, et al. Micrometastases and survival in stage II colorectal cancer. $N$ Engl J Med. 1998;339:223-8.

9. Shivers SC, Wang X, Li W, Joseph E, Messina J, Glass LF, et al. Molecular staging of malignant melanoma: correlation with clinical outcome. JAMA. 1998;280:1410-5.

10. Bostick PJ, Morton DL, Turner RR, Huynh KT, Wang HJ, Elashoff R, et al. Prognostic significance of occult metastases detected by sentinel lymphadenectomy and reverse transcriptase-polymerase chain reaction in early-stage melanoma patients. J Clin Oncol. 1999;17:3238-44.

11. Ooka M, Sakita I, Fujiwara Y, Tamaki Y, Yamamoto H, Aihara T, et al. Selection of mRNA markers for detection of lymph node micrometastases in breast cancer patients. Oncol Rep. 2000;7:561-6.

12. Mori M, Mimori K, Inoue H, Barnard GF, Tsuji K, Nanbara S, et al. Detection of cancer micrometastases in lymph nodes by reverse transcriptase-polymerase chain reaction. Cancer Res. 1995;55:3417-20.

13. Godfrey TE, Raja S, Finkelstein SD, Gooding WE, Kelly LA, Luketich JD. Prognostic value of quantitative reverse transcriptionpolymerase chain reaction in lymph node-negative esophageal patients. Clin Cancer Res. In press.

14. Godfrey TE, Kim SH, Chavira M, Ruff DW, Warren RS, Gray JW, et al. Quantitative mRNA expression analysis from formalin-fixed, paraffin-embedded tissues using $5^{\prime}$ nuclease quantitative RT-PCR. $J$ Mol Diagn. 2000;2:84-91. 
15. Gibson UE, Heid CA, Williams PM. A novel method for real time quantitative RT-PCR. Genome Res. 1996;6:995-1001.

16. Heid CA, Stevens J, Livak KJ, Williams PM. Real time quantitative PCR. Genome Res. 1996;6:986-94.

17. Raja S, Luketich JD, Kelly LA, Ruff DW, Godfrey TE. Increased sensitivity of one-tube, quantitative RT-PCR. Biotechniques. 2000;29: 702, 704, 706 .

18. Tassone F, Hagerman RJ, Taylor AK, Gane LW, Godfrey TE, Hagerman PJ. Elevated levels of FMR1 mRNA in carrier males: a new mechanism of involvement in the fragile-X syndrome. Am J Hum Genet. 2000;66:6-15.

19. PE Applied Biosystems. User bulletin No. 2. ABI Prism 7700 Sequence Detection System: relative quantitation of gene expression. Norwalk (CT): Perkin Elmer; 1997.

20. DeLong ER, DeLong DM, Clarke-Pearson DL. Comparing the areas under two or more correlated receiver operating characteristic curves: a nonparametric approach. Biometrics. 1988;44:837-45.

21. Heagerty PJ, Lumley T, Pepe MS. Time-dependent ROC curves for censored survival data and a diagnostic marker. Biometrics. 2000;56: 337-44.

22. Walsh TN, Noonan N, Hollywood D, Kelly A, Keeling N, Hennessy TP. A comparison of multimodal therapy and surgery for esophageal adenocarcinoma. $N$ Engl J Med. 1996;35:462-7.

23. Hennequin C, Gayet B, Sauvanet A, Blazy A, Perniceni T, Panis Y, et al. Impact on survival of surgery after concomitant chemoradiotherapy for locally advanced cancers of the esophagus. Int J Radiat Oncol Biol Phys. 2001;49:657-64.

24. Whitehair JG, Griffey SM, Olander HJ, Vasseur PB, Naydan D. The accuracy of intraoperative diagnoses based on examination of frozen sections: a prospective comparison with paraffin-embedded sections. Vet Surg. 1993;22:255-9.

25. Jannink I, Fan M, Nagy S, Rayudu G, Dowlatshahi K. Serial sectioning of sentinel nodes in patients with breast cancer: a pilot study. Ann Surg Oncol. 1998;5:310-4.

26. Karalak A, Homcha-Em P. Occult axillary lymph node metastases discovered by serial section in node-negative breast cancer. J Med Assoc Thai. 1999;82:1017-9.

\section{Discussion}

Dr Jack A. Roth (Houston, Tex). Raja and colleagues are to be congratulated for this novel and important presentation. I am frequently asked why thoracic surgeons should be familiar with the molecular biology of cancer. This interesting presentation provides an answer to that question: molecular diagnostics and therapeutics will be increasingly important tools in the management of patients with thoracic cancers. If we as surgeons are to play a significant role in the management of these cases, we need to be on the cutting edge of this technology.

This presentation describes an important advance in the use of cancer gene expression for the staging of esophageal cancer. To use this information clinically, it must be available rapidly, which is made possible by a technical advance in instrument design. Realistically, Raja and colleagues were able to show about a 10fold difference between lymph nodes with no evidence of metastases and lymph node cells expressing levels of CEA, which is also expressed at high levels by esophageal cancer cells. This difference is likely to be clinically useful.

An important issue raised by this study is the biologic behavior and significance of the micrometastases detected by this technique. Does the expression of a marker such as this really correlate with the presence of metastatic cancer cells? Dr Raja, are you planning a larger study to address this?

Some technical points could also be better addressed. CEA is not a highly tumor-associated protein and may, for example, be expressed by normal cells from smokers. Raja and colleagues now have many oncogene and tumor suppressor gene targets from which to choose. Dr Raja, have you expanded this study to include other tumor markers?

The number of patients in the study was small, and no indication of the variability of their triplicate samples is provided. How reproducible is the assay? Although the 30 minutes reported by the authors is faster than a frozen-section analysis at our institution, information showing the actual times needed to perform the assay would be useful and could convince impatient surgeons that this assay really can be used in the operating room.

The lower sensitivity of the frozen-section analysis is balanced by the reduced specificity of the RT-PCR assay, and the overall accuracies of the two procedures are therefore really similar. Thus, it is not clear that the additional information gained is costeffective. The value of this technique to assess nodal positivity in the operating room may have limited utility if, as for many thoracic cancers, a complete resection can be performed, surgery would proceed in that instance despite the presence of nodal metastases. However, this technique might be useful for assessing surgical margins. Dr Raja, have you used the technique for that purpose?

Use of this technique with fine-needle aspirate cytologic examination also might greatly improve staging accuracy. However, I believe that the real value of this technique is in rapidly establishing the molecular profile of the cancer. Rapid determination of molecular genetic profiles of the tumor will enable genetic lesions in cancer, such as overexpression of p53 or epidermal growth factor or angiogenic factors, to be specifically targeted with induction therapy. The first restoration of tumor suppressor gene function in lung cancer was reported 5 years ago, and now this treatment has been evaluated in many patients and has become a clinical reality. Genetically targeted therapies will become important adjuncts to surgery during the next 5 years, and genetic testing will be important in determining prognosis and therapy. Raja and colleagues are to be congratulated for leading us into the future.

Dr Raja. Regarding your first question about larger studies, since the time of this work we have been collecting tissues to be able to ultimately look at a much larger population of patients. We have not reached the accrual limit, and we therefore have not done the assays on those samples yet, but we are in the process of collecting the samples.

In answer to your second query, CEA was one of many markers that we had investigated, the others in the context of esophageal cancer being cytokeratin 19 and Mucin-1. CEA appeared to be the most sensitive and specific of these markers. Cytokeratin 19 was detected by immunohistochemical staining in mesothelium by our group, and because the samples were archived and we had no control over some of the tissues that were included, we could make sure that our blocks did not have esophageal tissue or epithelial cells but could not be certain that mesothelium was not present. We therefore excluded the other markers in this study. We are expanding our bank of molecular markers and molecular targets, and at the moment we have not used that in esophageal cancer.

To address your question about reproducibility, our assay is highly reproducible. We run a calibrator sample (the same RNA sample) along with every run in every experiment. The SD for this from run to run, for 13 runs, is 0.14 of the cycle, and the range is 
0.43 , which is significantly less than a 2 -fold difference. I think that this reproducibility has been lacking in the traditional standard RT-PCR, and that it is being addressed by quantitative RTPCR is what will ultimately be important in creating a clinical assay. The actual times to make this entire assay possible in our hands have been less than 25 minutes, with the RNA extraction being at 5 to 6 minutes.

In terms of the sensitivity and specificity of pathologic examination versus RT-PCR, we found that according to our survival curves this assay was similar to pathologic analysis, because a subset of patients have N1 disease and still survive. Looking at our fresh-tissue data, however, there seems to be a much better separation between the positive nodes and the negative nodes. So we believe that this assay, in combination with a multimarker approach, should resolve any issues of slightly decreased specificity that we are seeing in our study.

We are currently investigating the potential for verifying surgical margins. This approach is complicated by the fact that you need to find specific markers that are present in the tumor but not in the tissue from which it originated. DNA-based markers may provide an answer.

To answer the final question about the molecular profiling of these tumors, our work at the moment is a proof of principle. We have shown that the assay can be done in a rapid fashion. Once the assay is developed, modification to include other molecular targets, both DNA and RNA, should be quick to follow.

\section{Notice from the American Board of Thoracic Surgery regarding trainees and candidates for certification who are called to military service related to the war on terrorism}

The Board appreciates the concern of those who have received emergency call to military service. They may be assured that the Board will exercise the same sympathetic consideration as was given to candidates, in recognition of their special contributions to their country, during the Viet Nam conflict and the Persian Gulf conflict with regard to applications, examinations, and interruption of training. If you have any questions about how this might affect you, please call the Board office at 847-475-1520.

Peter C. Pairolero, MD

The American Board of Thoracic Surgery 